\title{
Collagen and Elastic Fibers in Odontogenic Entities: Analysis Using Light and Confocal Laser Microscopic Methods
}

\author{
Sabrina P. Moure ${ }^{1}$, Vinicius C. Carrard ${ }^{1}$, Isabel S. Lauxen ${ }^{1}$, Pedro Paulo A. Manso ${ }^{2}$, \\ Marcia G. Oliveira ${ }^{1}$, Manoela D. Martins ${ }^{1}$, Manoel Sant'Ana Filho ${ }^{1, *}$ \\ 'Oral Pathology, School of Dentistry, Universidade Federal do Rio Grande do Sul, Porto Alegre, Brazil \\ ${ }^{2}$ Pathology Department, Instituto Oswaldo Cruz - Fiocruz, Rio de Janeiro, Brazil
}

\begin{abstract}
Dentigerous cyst (DC) and keratocystic odontogenic tumor (KOT) are odontogenic lesions arising from epithelial elements, such as those observed in dental follicles (DF), that have been part of the tooth forming apparatus. These lesions show different clinical and histological characteristics, as well as distinct biological behavior. This study aimed to qualify and quantify collagen and elastic fibers by means of histochemical techniques with light and confocal laser microscopic methods in three odontogenic entities. Eleven DF, 13 DC ( $n=10$ with inflammation, n=3 without inflammation) and $13 \mathrm{KOT}$ were processed to the following techniques: Hematoxylin and Eosin, Masson's Trichrome, Picrosirius, Direct Blue, and Orcein. DF and DC without inflammation exhibited collagen with similar characteristics: no parallel pattern of fiber orientation, thick fibers with dense arrangement, and absence of distinct layers. A comparison between DC with inflammation and KOT revealed similar collagen organization, showing distinct layers: thin collagen fibers with loose arrangement near the epithelium and thick fibers with dense arrangement in distant areas. The only difference found was that KOT exhibited a parallel collagen orientation in relation to the odontogenic epithelia. It may be suggested that the connective tissue of DC is a reactive tissue, inducing an expansive growth associated with fluid accumulation and inflammatory process, which in turn may be present as part of the lesion itself. In KOT, loosely arranged collagen may be associated with the behavior of the neoplastic epithelium.
\end{abstract}

Keywords: Dental follicle, dentigerous cyst, keratocystic odontogenic tumor, extracellular matrix, confocal laser microscopy.

\section{INTRODUCTION}

Dental follicle (DF) is a structure that surrounds unerupted teeth and is composed of fibrous connective tissue that contains remnants of odontogenic epithelium with proliferative potential [1-3]. The proliferative potential of odontogenic epithelium in DF is not completely clear. In addition, the origin of some odontogenic cysts and tumors is attributed to this epithelium [4-6]. The most prevalent of these pathologies are dentigerous cysts (DCs) and keratocystic odontogenic tumors (KOTs) [6-9]. DC and KOT are odontogenic lesions arising from epithelial elements that have been part of the tooth forming apparatus. Although presenting a cystic appearance, DC and KOT show different clinical and histological characteristics, as well as distinct biological behavior [10]. KOTs have a relatively higher recurrence rate when compared with odontogenic cysts [7] and demonstrate locally aggressive behavior [6]. Several studies have been carried out in order to establish what drives the distinct features of KOT [10-14]. Most studies have focused on the evaluation of the proliferative activity in the epithelial component of these lesions $[8,15,16]$. KOT has shown increased proliferative activity when compared to DC and other cystic lesions [16].

*Address correspondence to this author at the Faculdade de Odontologia UFRGS Rua Ramiro Barcelos, 2492 / 503, Porto Alegre, RS. Brazil $90035-$ 003; Tel: +55 51 3308-5011; Fax: +55 51 3308-5023;

E-mail:manoel@ufrgs.br
The role of the extracellular matrix (ECM) in the pathogenesis and behavior of epithelial odontogenic lesions has been demonstrated in some publications $[8,9,17,18]$, suggesting that these lesions could be dependent on epithelial-mesenchymal interactions $[9,17,19-24]$. The ECM is a dynamic, intricate network of macromolecules that plays an important role in the maintenance of a correct microenvironment for basic cell functions, such as celladhesion, proliferation, and differentiation [25-28]. The major components of ECM are collagens, proteoglycans, and glycoproteins [28, 29]. The fibrous components of the ECM are classically divided into three fiber types: collagen, reticular, and elastic fibers. Some studies have addressed the role of ECM components in epithelial odontogenic lesions, especially the collagen component of DF, DC, and KOT, and discrepancies were observed [1, 2, 9, 18, 30,31]. Other ECM components, such as fibronectin, laminin, and tenascin have also been studied. The presence and distribution of elastic system fibers, which include oxytalan fibers, have been described in some tissues and lesions [32, 33]. However, there are no available data in the literature regarding odontogenic lesions.

Based on these facts, the aim of the present study was to concomitantly qualify and quantify the arrangement and distribution of collagen and elastic system fibers in three odontogenic entities, namely: dental follicle, dentigerous cyst, and keratocystic odontogenic tumor. 


\section{MATERIALS AND METHODOLOGY}

\section{Selection and Preparation of Samples}

This study was approved by the Research Ethics Committee of the School of Dentistry, Universidade Federal do Rio Grande do Sul (UFRGS), Brazil. The material was retrieved from the files of the Oral Pathology Laboratory, UFRGS School of Dentistry, Porto Alegre, Brazil. The study sample was composed of 11 sections of DF, 13 DC $(n=10$ with inflammation, $n=3$ without inflammation), and 13 KOT. None of the DF and KOT specimens showed evidence of inflammation. The specimens were selected by revision of hematoxylin and eosin (HE)-stained slides, and diagnosis was based on clinical description, radiographic and histological features, according to the criteria proposed by the WHO classification [34].

Four 3-micrometer sections were obtained from each paraffin block and processed to the following staining techniques: (a) Masson's Trichrome (MT); (b) phosphomolybdic acid-picrosirius red modified for confocal laser microscopy (PMA-PSR) [35]; (c) direct blue (DB) [36]; and (d) orcein (OX-OR) [37]. All staining procedures were performed at the Department of Pathology of Instituto Oswaldo Cruz (IOC - Fiocruz), Rio de Janeiro, Brazil.

Images of MT-stained specimens were captured using a video camera (Q-Color5 ${ }^{\mathrm{TM}}$; Olympus Latin America, Inc., Miami, FL, USA), coupled to a binocular microscope (CX41RF; Olympus Latin America, Inc., Miami, FL, USA) and a PC Dimension 5150 (Dell, Porto Alegre, RS, Brazil), and then imported to and recorded by QCapture ${ }^{\circledR}$ software, version 2.81 (QImaging Co., Surrey, BC, Canada). Since specimens had different sizes, the number of microscopic fields digitized for each sample ranged from 3 to 10 . The fields were recorded at $\times 100$ and $\times 400$ magnification.

Images of PMA-PSR-, DB-, and OX-OR-stained specimens were obtained using a Zeiss LSM 510 META confocal laser scanning microscope (Zeiss, Oberkochen, BadenWürttemberg, Germany) with $\mathrm{HeNe}$ laser (543 nanometers) and LP 560 filter. Two fields were obtained from each slide at $x 100$ and $x 400$ of the original magnification. All images were recorded by + LSM Image Browser ${ }^{\circledR}$ software, version 3.5 (Zeiss, Oberkochen, Baden-Württemberg, Germany), which was configured to show collagen fibers in white and gray tones, whereas interstitial space and epithelial tissue were displayed as black areas.

The same microscopic fields were selected in each staining technique. In DF specimens, epithelial nests surrounded by connective tissue areas were analyzed. In DC and KOT, fields including epithelial tissue and the adjacent connective tissue were evaluated.

The examiner had been calibrated in advance of the experimental assessments under the supervision of an experienced pathologist. Intra-examiner calibration was performed by means of a second analysis of 1 out of every 10 fields observed, by applying the intraclass correlation coefficient ( $\mathrm{p}$ $<0.01)$ and the kappa coefficient test ( $p>0.7)$ to determine the degree of agreement for quantitative and qualitative analyses, respectively. The examiner was blind to which group the images belonged.

\section{MT Analysis}

A qualitative description of the morphological aspects of collagen orientation and arrangement observed on images of MT-stained specimens was performed. A semiquantitative analysis (score system) was performed according to the criteria and scores described below.

\section{Collagen Fiber Orientation}

This criterion analyzed collagen fiber orientation in relation to the epithelial component. The analysis was performed at $\mathrm{x} 100$ and $\mathrm{x} 400$ magnification. Each case was classified according to the fiber orientation pattern as 1: No parallel; or 2: Parallel.

\section{Arrangement of Collagen Fibers}

The organization of collagen fibers was evaluated in areas near and distant from the epithelial component. The analysis was performed at $\mathrm{x} 100$ and $\mathrm{x} 400$ magnification. Each case was classified as 1: Loose bundles of collagen fibers (loosely arranged and interwoven in all directions); or 2: Dense (well-defined organization with orderly organized collagen fibers forming collagen lamellae).

\section{Fiber Organizatio, Organization}

The distribution of collagen fibers was evaluated along the connective tissue. The analysis was performed at $x 100$ magnification. Each case was classified as 1: Absent (collagen organization was similar throughout the connective tissue displaying one single collagen layer); or 2: Present (collagen fibers were arranged in two distinct layers).

\section{PMA-PSR Analysis}

A qualitative analysis of the morphological aspects of PMA-PSR-stained collagen fibers was performed using confocal laser microscopy. Quantitative analysis of the collagen component was performed based on images of each specimen using Image-Pro ${ }^{\circledR}$ Plus software, version 5.1 (Media Cybernetics, Inc., Silver Spring, MD, USA), in which the white and gray tone frequency distribution of pixels by image was converted into a histogram. White and gray pixels were added up and divided by the total number of pixels (white, gray, and black), resulting in the proportion of collagen fibers for each microscopic field. Mean collagen fiber proportion was then calculated for each case.

\section{DB and OX-OR Analysis}

Images of DB-stained specimens were used to identify elastic fibers, and OX-OR images to identify oxytalan fibers. Skin was a positive control for DB staining and blood vessels for OX-OR staining. The descriptive analysis was based on the assessment of either presence or absence of these fibers.

\section{Statistical Analysis}

The semiquantitative analysis of MT-stained specimens compared three distinct groups (DF, DC, KOT) according to collagen fiber orientation, arrangement of collagen fibers, and distinct layers of collagen fiber organization using the Kruskal-Wallis test. Multiple comparisons were performed 

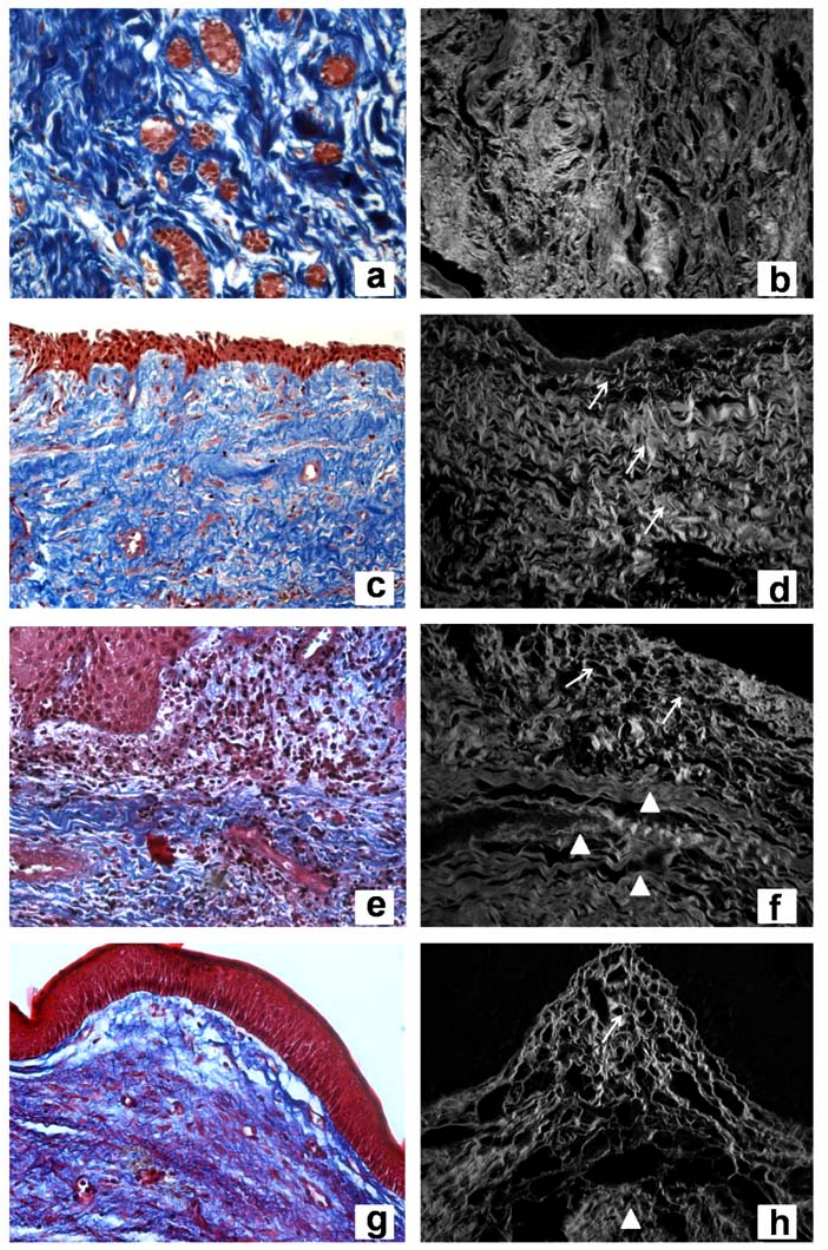

Fig. (1). Masson's Trichrome of dental follicle (a), dentigerous cyst without inflammation (c), dentigerous cyst with inflammation (e), and keratocystic odontogenic tumor (g) (original magnification, $\mathrm{x} 400$ ). Confocal laser microscopy of Picrosirius red staining of dental follicle (b), dentigerous cyst without inflammation (d), dentigerous cyst with inflammation (f), and keratocystic odontogenic tumor (h) (original magnification, $\mathrm{x} 400$ ).

using Dunn's test whenever any differences were observed between groups. Quantitative analysis of variance (ANOVA) was used to compare amounts of collagen fiber in PMAPSR-stained specimens. Significance level was set at $5 \%$ (p $<$ 0.05). All statistical analyses were performed using GraphPad Prism, version 4.0 (GraphPad Software, Inc., San Diego, CA, USA).

\section{RESULTS}

\section{MT Analysis}

\section{Qualitative and Semiquantitative}

Overall, DF specimens exhibited collagen fibers not arranged in parallel bundles (no parallel pattern) in relation to islands or a single layer of odontogenic epithelium. The collagen component was densely arranged and uniformly distributed throughout the connective tissue (Fig. 1a).

DC, regardless of the presence or absence of inflammation, showed collagen fibers without parallel disposition in relation to the epithelial layer (Fig. 1c, 1e). The main difference observed was that DC without inflammation had loosely arranged collagen fibers throughout the specimen (Fig. 1c), whereas DC with inflammation showed two distinct layers of collagen organization. The latter exhibited a loose arrangement near the epithelium and dense connective tissue in other parts of the lesion (Fig. 1e).

KOT showed collagen fibers oriented in a parallel pattern and loosely arranged near the epithelial lining. Two distinct layers of collagen could be observed along the connective tissue (Fig. 1g).

The results of semiquantitative MT analysis performed in all three odontogenic entities under study are described in Table 1.

Significant differences were observed between DF, DC, and KOT according to the score analysis of orientation, arrangement, and presence of distinct layers of collagen fibers $(\mathrm{p}<0.05)$.

In the analysis of collagen fiber orientation, KOT exhibited different patterns of fiber orientation when compared to DF and DC ( $<<0.05)$. KOT showed a parallel collagen orientation in relation to the odontogenic epithelia, whereas DF and DC did not show a parallel pattern of fiber orientation. This difference in collagen fiber orientation between KOT and DC was observed both in the presence and absence of inflammation $(\mathrm{p}<0.05)$.

The arrangement of DF and KOT collagen fibers was shown to be significantly different $(\mathrm{p}<0.05)$. DF connective tissue showed a dense arrangement, whereas KOT exhibited a loose collagen arrangement. DC with inflammation exhibited a loose arrangement, while DC without inflammation showed a dense organization. Comparing DC subtypes, a significant difference in collagen arrangement was observed between DC without inflammation and KOT $(\mathrm{p}<0.05)$ and DC with inflammation and DF ( $\mathrm{p}<0.05)$.

When analyzing distinct layers of collagen fiber organization, DF exhibited a distinct pattern compared to DC with inflammation and KOT $(\mathrm{p}<0.05)$.

\section{PMA-PSR Analysis}

\section{Qualitative}

DF exhibited thick fibers with dense and interwoven arrangement surrounding the odontogenic epithelial island (Fig. 1b). DC without inflammation showed thick fibers throughout the connective tissue wall (Fig. 1d), whereas DC with inflammation showed a thin collagen fiber layer near the epithelium associated with the inflammatory infiltrate (Fig. 1f). The area without inflammation exhibited thick fibers arranged in dense bundles (Fig. 1f). KOT showed thin fibers loosely arranged near the epithelium (Fig. 1h) and thick fibers densely arranged in a distant area (Fig. 1f).

\section{Quantitative}

Quantitative analysis of the proportion of collagen fibers revealed no differences between groups $(\mathrm{p}=0.3122)$. 
Table 1. Semiquantitative Analysis of Masson's Trichrome Staining of Odontogenic Entities Based on Morphological Characteristics of Collagen Fibers

\begin{tabular}{|c|c|c|c|c|c|c|c|}
\hline \multirow{2}{*}{ Entities / Collagen } & \multicolumn{2}{|c|}{ Orientation } & \multicolumn{2}{c|}{ Arrangement } & \multicolumn{2}{c|}{ Distinct Layers } \\
\cline { 2 - 7 } & Parallel & No Parallel & Loose & Dense & Absent & Present \\
\hline \hline \multirow{2}{*}{$\begin{array}{c}\text { DF } \\
(\mathbf{n = 1 1})\end{array}$} & & $1(9.1 \%)^{\mathrm{a}}$ & $10(90.9 \%)$ & $4(33.3 \%)^{\mathrm{a}}$ & $7(66.7 \%)$ & $11(100 \%)^{\mathrm{a}}$ & 0 \\
\hline \multirow{2}{*}{$\begin{array}{c}\mathbf{D C} \\
(\mathbf{n = 1 3})\end{array}$} & without inflammation $(\mathrm{n}=3)$ & $0^{\mathrm{a}}$ & $3(100 \%)$ & $1(33.3 \%)$ & $2(66.7 \%)^{\mathrm{a}}$ & $3(100 \%)^{\mathrm{a}}$ & 0 \\
\cline { 2 - 8 } & $\begin{array}{c}\text { with inflammation } \\
(\mathrm{n}=10)\end{array}$ & $0^{\mathrm{a}}$ & $10(100 \%)$ & $9(90.9 \%)^{\mathrm{b}}$ & $1(9.1 \%)$ & $0^{\mathrm{b}}$ & $10(100 \%)$ \\
\hline $\begin{array}{c}\text { KOT } \\
(\mathbf{n = 1 3})\end{array}$ & & $12(92.3 \%)^{\mathrm{b}}$ & $1(7.7 \%)$ & $11(84.6 \%)^{\mathrm{b}}$ & $2(15.4 \%)$ & $2(15.4 \%)^{\mathrm{b}}$ & $11(84.6 \%)$ \\
\hline
\end{tabular}

$\mathrm{DC}=$ dentigerous cysts; $\mathrm{DF}=$ dental follicles; $\mathrm{KOT}=$ keratocystic odontogenic tumors.

${ }^{\mathrm{a}, \mathrm{b}}$ Values followed by the same letter are not significantly different from each other.

\section{DB and OX-OR Analysis}

No elastic or oxytalan fiber labeling was observed in the studied entities.

\section{DISCUSSION}

The present study performed a qualitative and quantitative analysis of collagen and elastic system fibers, which are fibrous components of the ECM, in three odontogenic entities. All odontogenic entities analyzed revealed a fibrous component associated with an epithelial tissue. It is well known that fiber organization and epithelial-mesenchymal interactions lead to different biological behaviors.

Our results demonstrated that DF, DC, and KOT exhibited absence of elastic system fibers and a similar amount of collagen fibers. However, collagen fibers showed different morphological features regarding orientation and arrangement. The quality and organization of collagen fibers significantly affect the tensile strength of a connective tissue and consequently its capacity to support tissues and organs [3840]. Collagen is the major stromal component of DF, DC, and KOT and was verified in this study by two-dimensional MT staining analysis and three-dimensional confocal laser microscopy with PMA-PSR staining analysis. These techniques allowed us to detect not only the presence but also characteristics of collagen fibers. Quantitative analyses of collagen fibers, especially using the picrosirius-polarization method, have been described in the literature [41-43]. However, the use of a confocal laser microscope may improve this analysis by providing higher image resolution [41].

In the present study, collagen in DF and DC without inflammation was found to have similar morphological characteristics, showing no parallel pattern of collagen fiber orientation and absence of distinct layers at MT analysis. On the other hand, DC with inflammation and KOT exhibited comparable collagen organization, showing loose arrangement and presence of distinct layers at MT analysis. Under confocal laser microscopy, collagen fibers were thin and loosely arranged near the epithelium and thick and densely arranged in distant areas. The only morphological difference observed between KOT and DC with inflammation was that KOT exhibited a parallel collagen orientation in relation to the odon- togenic epithelia, whereas DC showed no parallel pattern of fiber orientation.

When comparing our results regarding morphological aspects and function of collagen fibers, DF and DC without inflammation appeared to have dense connective tissue, which is necessary for architecture stabilization of dental tissue surrounding unerupted teeth. The connective tissue responds to low-intensity stimuli by maintaining the production of dense and organized collagen. The presence of dense fibrous connective tissue in DC capsules has also been reported in previous studies [2, 4]. In contrast, DC with inflammation and KOT showed loose connective tissue, which could be interpreted as pathologic or poor packing of collagen fibers as a result of the development of inflammatory and tumor processes. One may speculate that in DC this characteristic is attributed to the inflammatory infiltrate and in KOT it is associated with changes induced by neoplastic epithelia, promoting ECM degradation and infiltrative growth.

Although DCs are classified as developmental lesions, the present study, as well as previous reports in the literature, found inflammation in the connective tissue wall in several cases [31]. Benn et al. [44] proposed the existence of two types of DC: one developmental and the other inflammatory in nature. It is well known that the proliferative capacity of epithelial cells is an indispensable ingredient for cyst formation [45]. This capacity was reinforced by da Silva Baumgart et al. [46], who assessed cells of odontogenic epithelial nests and of the reduced epithelium of the enamel organ present in dental follicles and supported their involvement in the formation of cysts and tumors. If tooth eruption is capable of generating an inflammatory process [44], pro-mitotic chemotactic inflammatory factors present in the connective tissue may reach the reduced enamel epithelium in dental follicles, whose growth factor receptors are located in the membrane and cytoplasm [46], giving rise to DC. DCs with presence of inflammatory infiltrate in the connective tissue were associated with a shift from a typical epithelium into a hyperplastic non-keratinizing stratified squamous epithelium, in addition to a direct influence on the organization and thickness of collagen fibers. DC without inflammation revealed dense 
collagen organization similar to DF. In cases with inflammation, loose collagen networks were observed near the epithelium and dense networks in the periphery. Inflammation within the DC wall may alter the microenvironment so that the collagen component becomes more similar to that found in KOT. However, the stimuli that lead to such characteristic are different in these lesions: inflammation is responsible for changes in DC and tumorigenesis in KOT [45].

In DC with inflammation, the main aspect likely to induce changes in collagen organization is the presence of plasma exudates in the interstitium. These exudates are rich in inflammatory mediators, such as IL- $1 \beta$, TNF- $\alpha$, and prostaglandins, which regulate ECM production and degradation as well as bone resorption. In other chronic inflammatory conditions, such as chronic gingivitis and periodontitis, similar connective tissue destruction occurs in response to inflammation. It is believed that, in these lesions, stromal cells, such as resident fibroblasts or inflammatory cells, produce and release matrix metalloproteinases (MMPs) and cytokines (IL-1 $\beta$ and TNF- $\alpha$ ) that amplify the inflammatory response by stimulating collagenase activity, which leads to loosely arranged collagen fibers in such areas.

The collagen organization observed in KOT in this study is in agreement with that observed by other authors $[9,18]$, who reported that collagen fibers found in the fibrous KOT wall were as structurally disorganized as those observed in odontogenic tumors, but different from those observed in other types of cysts. This loose and parallel pattern of collagen arrangement observed in KOT may be useful to facilitate the separation of the epithelial lining from the underlying connective tissue wall, the presence of satellite cysts, and the invasive potential of the lesion, which are important characteristics of this tumor.

Another explanation for the different patterns of collagen observed in this study may be related to the type of collagen present in each entity. A quantitative analysis of the collagen component by confocal laser microscopy revealed that all entities had similar collagen content. However, the techniques used in this study did not separate the types of collagen. Therefore, although the amount of collagen was similar, the types of collagen could have been different.

Connective tissue stroma has been suggested to play an important role in the pathogenesis of KOT in several publications [9, 18, 45]. Hirshberg et al. [18] suggest that connective tissue stroma of KOT could be regarded not just as a structural support of the cyst wall, but as a functional part of the lesion. Our results showed that the collagen component of KOT stroma has a different arrangement compared to other odontogenic entities. However, further studies are needed to indicate that stroma is a functional part of the tumor, especially regarding MMP activity.

\section{CONCLUSION}

Assuming that DF is as a normal odontogenic structure and taking into account the results described here and the above-mentioned properties of collagen fibers, we suggest that DC connective tissue is a reactive tissue. Its role appears to be that of maintaining an expansive growth associated with fluid accumulation and inflammatory process, which in turn may be present as part of the lesion. On the other hand, in KOT, loosely arranged collagen may be related to the neoplastic behavior of the lesion.

\section{ACKNOWLEDGEMENTS}

The authors would like to thank Dr. Marcelo PelajoMachado and Dr. Luzia Caputo from the Department of Pathology at Instituto Oswaldo Cruz (IOC - Fiocruz, RJ, Brazil) for making resources available for special staining techniques and confocal laser microscopy. This study received financial support from FAPERGS (Fundação de Amparo à Pesquisa do Estado do Rio Grande do Sul; PROAP 04/2005, Process no. 0410882) and CNPq (Conselho Nacional de Desenvolvimento Científico e Tecnológico).

\section{REFERENCES}

[1] Kim J, Ellis G. Dental follicular tissue: misinterpretation as odontogenic tumors. J Oral Maxillofac Surg 1993; 51: 762-7.

[2] Rakprasitkul S. Pathologic changes in the pericoronal tissues of unerupted third molars. Quintessence Int 2001; 32: 633-8.

[3] Curran A, Damm D, Drummond J. Pathologically significant pericoronal lesions in adults: histopathologic evaluation. J Oral Maxillofac Surg 2002; 60: 613-7.

[4] Saraçoğlu U, Kurt B, Günhan Ö, Güven O. MIB-1 expression in odontogenic epithelial rests, epithelium of health oral mucosa and epithelium of selected odontogenic cysts: an immunohistochemical study. Int. J Oral Maxillofac Surg 2005; 34: 432-43.

[5] Ide F, Obara K, Yamada H, et al. Hamartomatous proliferations of odontogenic epithelium within the jaws: a potential histogenetic source of intraosseous epithelial odontogenic tumors. J Oral Pathol Med 2007; 36: 229-35

[6] Vered M, Shohat I, Buchner A, Dayan D. Myofibroblasts in stroma of odontogenic cysts and tumors can contribute to variations in the biological behavior of lesions. Oral Oncol 2005; 41: 1028-33.

[7] Poomsawat S, Punyasingh J, Weerapradist W. Expression of basement membrane components in odontogenic cysts. Oral Dis 2006; 12: 290-6.

[8] Oliveira M, Lauxen I, Chaves A, Rados P, Sant'Ana Filho M. Immunohistochemical analysis of the patterns of p53 and PCNA expression in odontogenic cystic lesions. Med Oral Patol Oral Cir Bucal 2008; 13: e275-80.

[9] Hirshberg A, Sherman A, Buchner A, Dayan D. Collagen fibres in the wall of odontogenic keratocysts: a study with picrosirius red and polarizing microscopy. J Oral Pathol Med 1999; 28: 410-2.

[10] Ide F, Saito I. Many faces of odontogenic keratocyst. Oral Oncol 2003; 39: 204-5.

[11] Kimi K, Kumamoto H, Ooya K, Motegi K. Immunohistochemical analysis of cell-cycle- and apoptosis-related factors in lining epithelium of odontogenic keratocysts. J Oral Pathol Med 2001; 30: 4342.

[12] Kubota Y, Oka S, Nakagawa S, Shirasuna K. Interleukin-1alpha enhances type I collagen-induced activation of matrix metalloproteinase-2 in odontogenic keratocyst fibroblasts. J Dent Res 2002; 81: 23-7.

[13] Lam C, Leung C, Lee K, et al. Novel mutations in the PATCHED gene in basal cell nevus syndrome. Mol Genet Metab 2002; 76: 57 61.

[14] Shear M. The aggressive nature of the odontogenic keratocyst: is it a benign cystic neoplasm? Part II. Proliferation and genetic studies. Oral Oncol 2002; 38: 323-31.

[15] Mateus G, Lanza G, de Moura P, Marigo H, Horta M. Cell proliferation and apoptosis in keratocystic odontogenic tumors. Med Oral Patol Oral Cir Bucal 2008; 13: E697-702.

[16] Gadbail A, Chaudhary M, Patil S, Gawande M. Actual proliferating index and p53 protein expression as prognostic marker in odontogenic cysts. Oral Dis 2009; 15: 490-8.

[17] Wahlgren J, Maisi P, Sorsa T, et al. Expression and induction of collagenases (MMP-8 and -13) in plasma cells associated with bone-destructive lesions. J Pathol 2001; 194: 217-24. 
[18] Hirshberg A, Lib M, Kozlovsky A, Kaplan I. The influence of inflammation on the polarization colors of collagen fibers in the wall of odontogenic keratocyst. Oral Oncol 2007; 43: 278-82.

[19] Vedtofte P, Holmstrup P, Dabelsteen E. Human odontogenic keratocyst transplants in nude mice. Scand J Dent Res 1982; 90: 30614.

[20] Teronen O, Salo T, Laitinen J, et al. Characterization of interstitial collagenases in jaw cysts wall. Eur J Oral Sci 1995; 103: 141-7.

[21] Lukimaa P, Leppäniemi A, Hietanen J, Allemanni G. Zardi L. Features of odontogenesis and expression of cytokeratins and tenascin- $\mathrm{C}$ in three cases of extraosseous and intraosseous calcifying odontogenic cyst. J Oral Pathol Med 1997; 26: 265-72.

[22] da Silva M, de Sousa S, Corrêa L, Carvalhosa A, de Araújo V. Immunohistochemical study of the orthokeratinized odontogenic cyst: a comparison with the odontogenic keratocyst. Oral Surg Oral Med Oral Pathol Oral Radiol Endod 2002; 94: 732-7.

[23] Abrahão I, Martins M, Katayama E, Antoniazzi J, Segmentilli A, Marques M. Collagen analysis in human tooth germ papillae. Braz Dent J 2006; 17: 208-12.

[24] Harumi MS, Kerkis I, da Costa MC, Gomes C, Martins M, Marques M. Expression of extracellular matrix proteins in human dental pulp stem cells depends on the donor tooth conditions. J Endod 2010; 36: 826-31.

[25] Thesleff I, Vainio S, Jalkanen M. Cell-matrix interactions in tooth development. Int J Dev Biol 1989; 33: 91-7.

[26] Boudreau N, Jones P. Extracellular matrix and integrin signalling: the shape of things to come. Biochem J 1999; 339: 481-8.

[27] Jones P, Jones F. Tenascin-C in development and disease: gene regulation and cell function. Matrix Biol 2000; 19: 581-96.

[28] Ioachim E, Charchanti A, Briasoulis E, et al. Immunohistochemical expression of extracellular matrix components tenascin, fibronectin, collagen type IV and laminin in breast cancer: their prognostic value and role in tumour invasion and progression. Eur $\mathbf{J}$ Cancer 2002; 38: 2362-70.

[29] Chiquet-Ehrismann R, Hagios C, Schenk S. The complexity in regulating the expression of tenascins. Bioessays 1995; 17: 873-8.

[30] Browne R. The odontogenic keratocyst. histological features and their correlation with clinical behavior. Br Dent J 1971; 21: 249-59.

[31] Ahlfors E, Larsson A, Sjögren S. The odontogenic keratocyst: a benign cystic tumor? J Oral Maxillofac Surg 1984; 42: 10-9.

[32] Ushiki T. Collagen fibers, reticular fibers and elastic fibers: a comprehensive understanding from a morphological viewpoint. Arch Histol Cytol 2002; 65: 109-26.
[33] Staszyk C, Gasse H. Oxytalan fibres in the periodontal ligament of equine molar cheek teeth. Anat Histol Embryol 2004; 33: 17-22.

[34] Barnes L, Eveson W, Reichart P, Sidransky D. World health organization classification of tumors. Pathology \& Genetics Head and Neck Tumours. $1^{\text {st }}$ ed. Lyon: IARC Press 2005.

[35] Dolber P, Spach M. Conventional and confocal fluorescence microscopy of collagen fibers in the heart. J Histochem Cytochem 1993; 41: 465-9.

[36] de Brito-Gitirana L, Trindade A. Direct blue staining plus polarization microscopy: an alternative dye for polarization method for collagen detection in tissue sections. J Histotechnol 2000; 23: 1-3.

[37] Bancroft J, Stevens A. Theory and practice of histological techniques. $5^{\text {th }}$ ed. London: Churchill Livingstone 1996.

[38] Junqueira L, Montes G. Biology of collagen-proteoglycan interaction. Arch Histol Jpn 1983; 46: 589-629.

[39] Montes G, Junqueira L. The use of the picrosirius-polarization method for the study of the biopathology of collagen. Mem Inst Oswaldo Cruz 1991; 86: 1-11.

[40] Aumailley M, Gayraud B. Structure and biological activity of the extracellular matrix. J Mol Med 1998; 76: 253-65.

[41] Carvalho $\mathrm{H}$, Taboga $\mathrm{S}$. The applicability of hematoxylin-eosin staining plus fluorescence or confocal laser scanning microscopy to the study of elastic fibers in cartilages. Acad Sci Paris, Science de la vie/Life sciences 1996; 319: 991-6.

[42] Lenzi H, Kimmel E, Schechtman H, et al. Collagen arrangement in hepatic granuloma in mice infected with Schistosoma mansoni: dependence on fiber radiation centers. Braz J Med Biol Res 1999; 32: 639-43.

[43] Fung D, Ng G, Leung M, Tay D. Investigation of the collagen fibril distribution in the medial collateral ligament in a rat knee model. Connect Tissue Res 2003; 44: 2-11.

[44] Benn A, Altini M. Dentigerous cysts of inflammatory origin: a clinicopathologic study. Oral Surg Oral Med Oral Pathol Oral Radiol Endod 1996; 81: 203-9.

[45] Browne R. Pathogenesis of odontogenic cysts. In: Investigative pathology of the odontogenic cysts. $1^{\text {st }}$ ed. Boca Raton: CRC Press 1991.

[46] da Silva Baumgart C, Lauxen I, Sant'Ana Filho M, de Quadros O. Epidermal growth factor receptor distribution in pericoronal follicles: relationship with the origin of odontogenic cysts and tumors. Oral Surg Oral Med Oral Pathol Oral Radiol Endod 2005; 103: 240-5.

\footnotetext{
Received: January 18, 2011

Revised: April 25, 2011

Accepted: April 27, 2011

(C) Moure et al.; Licensee Bentham Open.

This is an open access article licensed under the terms of the Creative Commons Attribution Non-Commercial License

(http://creativecommons.org/licenses/by-nc/3.0/) which permits unrestricted, non-commercial use, distribution and reproduction in any medium, provided the work is properly cited.
} 